\title{
A New Depth Saliency Method Based on Selective Difference
}

\author{
Qiu-Xia CHEN, Li-Hua FU, Can-Can LI, Hao-Gang DING \\ Beijing University of Technology,Beijing, China \\ yuxiu_15@163.com,fulh@bjut.edu.cn,can1669103125@163.com,dinghaogang26@163.com
}

\begin{abstract}
Stereopsis plays an important role in the human visual system. In this paper, we propose a novel regional contrast based saliency method, which works on depth images based on selective difference. In this work, we measure the saliency of a region based on regional contrast and take depth difference in horizontal direction into consideration. Besides, center prior is used here to refine the initial saliency map. The proposed method can better reflect the influence of depth information on saliency detection, and the results are more in line with human vision.
\end{abstract}

\section{Introduction}

Human visual system can quickly obtain the information of interest regions from complex scenes [1]. Saliency analysis simulate the selective mechanism of human vision, which is widely used in the field of computer vision, including object detection and recognition, image retrieval, compression and retargeting. Saliency detection method can be divided into two parts: bottom-up(data-driven) and topdown(task-driven)[2]. In this paper, we focus on the bottomup saliency detection model.

The purpose of saliency detection is to simulate the human visual perception, and locate the most salient object rapidly and accurately. Saliency detection mainly depends on the collection of visual information and the extraction of features, previously, most of the saliency detection methods use color images as input, calculate the saliency value based on visual features such as color, shape, orientation, texture, etc[3-9]. When the salient object has at least one distinctive visual feature, the salient object can be detected as salient, on the contrary, lack of 3D spatial information, the detection result is not valid, as shown in Fig. 1(e) and 1(f).

Stereopsis provides a very useful cue for saliency detection[10], which can be used to identify salient objects with similar visual features. Recently, depth images has been used as the fourth channel combined with the existing 2D model to improve the saliency map [11-13,15]. Alternatively,

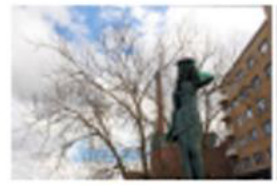

(a) Lftimage

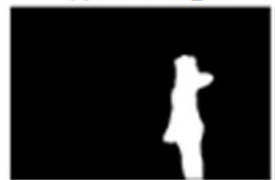

(d) Ground Truth

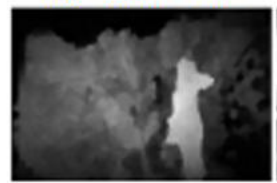

(g) $\operatorname{ACSD}[17,18]$

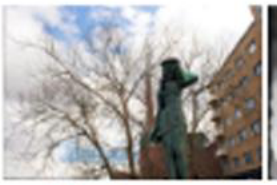

(b) Right image

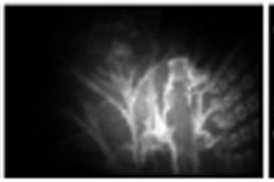

(e) PCA[21]

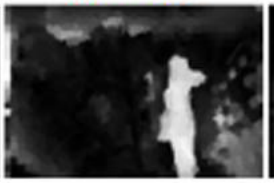

(h) $S[16]$

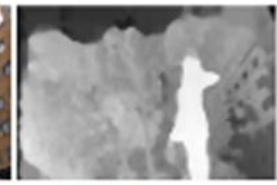

(c) Depth image

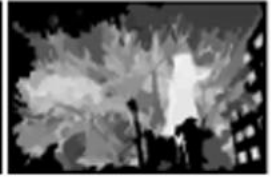

(f) $\mathrm{RC}[3]$

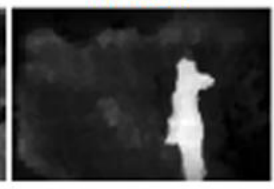

(i) Orrs
Fig. 1 Saliency maps generated by different methods This example shows that the saliency method based on depth information supplies a powerful cue for saliency detection.

the depth image is also used to construct the 3D layout[14]. To fully exploit the characteristics of depth information, Niu et al.[16] proposed a saliency detection model based on global disparity contrast and stereo photography domain knowledge, Ran et al.[17,18] proposed a depth-aware saliency method using anisotropic centersurround difference. In addition, a variety of priors[12,17] are used to refine the initial saliency map. Ran and Niu are both based on the basic hypothesis and characteristics of depth images: salient regions have a strong contrast with the background regions, therefore, it's easier to detect the most salient object based on depth images. This assumption 
ignores the large background area at the bottom of the depth image, which are always mistakenly detected as salient.

In this paper, we propose a novel depth saliency method to solve the problems mentioned above. Experiments show that the model can obtain the complete salient objects, at the same time, effectively weaken the saliency of background regions.

\section{Depth-aware Saliency Detection based on Selective Difference}

In this paper, we propose a depth contrast based saliency detection model. Firstly, we obtain the depth images and segment the color image into multiple image sub-regions. Then we measure the saliency of a region based on global contrast and consider the depth difference in horizontal direction. Besides, center prior is used to refine the initial saliency map. Finally, we obtain a binary image as the final saliency map.

\subsection{Pretreatment}

There are many devices used to obtain depth images, such as ToF(Time of Flight), Laser Rangefinders, Structured light scanner and so on. The optical flow method proposed by Sun et al.[19] is used here for easy access. As shown in Fig. 2(a) and 2(b) are respectively corresponding to the left and right eyes at the image, (c) corresponding to the depth map, obviously, the farther away the camera is, the brighter the pixel is, and vice versa.

In this work, we get the sub-regions of color images using SLIC(Simple Linear Iterative Clustering)[20] algorithm. Besides, Gaussian template is used to smooth the depth images for better accuracy.

\subsection{Saliency Detection based on Regional Contrast}

It is easier to attract people's attention in the area of the image, which have a larger contrast with the surrounding objects. Meanwhile, the spatial distance also plays a very important role, and the high contrast of the adjacent area makes it easier for a region to cause visual attention[3]. Cheng et al.[3] proposed a saliency method based on spatial relations and regional contrast. In this work, the RC(Region Contrast) method is improved here to calculate the saliency value based on depth information, which is calculated as follows:

$$
S_{G}\left(R_{i}\right)=\sum_{R_{k} \in C_{L}\left(R_{i}\right)} d\left(c_{i}, c_{k}\right) w\left(c_{i}, c_{k}\right)
$$

$S_{G}\left(R_{i}\right)$ represents the global selective difference of region $R_{i}$. In this work, we set $L$ as the neighborhood radius of $R_{i}, C_{L}\left(R_{i}\right)$ represents all the regions in the neighborhood radius of $R_{i}$. Considering the continuity of depth information, we take $c_{i}$ (the centroid of region $R_{i}$ ) to represent region $R_{i}$.

$d(p, q)=\left\{\begin{array}{c}d_{q}-d_{p}, d_{q}-d_{p}>0 \\ 0, \quad d_{q}-d_{p} \leq 0\end{array}\right.$

$d(p, q)$ represents depth contrast between pixels $p$ and $\mathrm{q}$, and $d_{p}, d_{q}$ respectively corresponding to the depth value of pixel $p$ and $\mathrm{q}$.
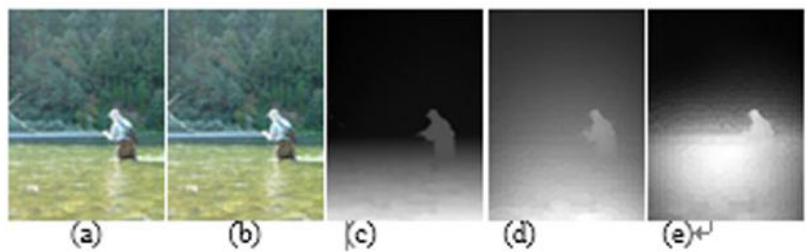

Fig. 2 Saliency map based on regional contrast. (a) Left image, (b) Right image, (c) Depth image, (d) $L$ is set to the image diagonal, (e) $L$ is set to three-third of the image diagonal.

$w(p, q)=e^{-D_{s}(p, q) / \sigma^{2}}$

Particularly, considering the influence of spatial distance between two pixels, we set $w(p, q)$ as the weight of the distance. $D_{s}(p, q)$ is the spatial distance between pixels $p$ and $q$, and $\sigma^{2}$ is assigned to control the spatial distance.

Fig. 2 shows the results of the saliency detection based on regional contrast. As shown in Fig. 2(e), the saliency map is more in line with the expectations and now $L$ is set to three-third of the image diagonal.

\subsection{Saliency Detection in horizontal direction}

Salient objects are usually surrounded by the background regions, which is extended to the whole range of the depth image, thus some background regions are mistakenly detected as salient. For example, as shown in Fig. 2(e), the water at the bottom of the image detected as salient areas, has a high contrast with respect to the other regions, thus it is detected as salient.

We observe that depth difference between pixels of each line is smooth in some background regions, such as the ground at the bottom of the image. To describe it in more detail, the depth difference of horizontal direction in the background regions is smooth, conversely, the depth difference is abrupt in areas with salient objects. Similar views are presented in [16], and the difference is that we take the selective difference, not the absolute difference, as the comparison result. According to this characteristic, a new detection method in horizontal direction is proposed to suppress the saliency of background regions and highlight 
the saliency of salient objects. In this work, we define the saliency as follows:

$S_{L}\left(R_{\mathrm{i}}\right)=\frac{\sum_{p \in R_{i}} d(p, B)}{n_{i}}$

$S_{L}\left(R_{i}\right)$ represents the local selective difference of region $R_{i} . n_{i}$ is the number of pixels of region $R_{i} . p$ is a pixel of region $R_{i}$.

$d(p, B)=\left\{\begin{array}{c}d_{r}^{B}-d_{p}, d_{r}^{B}-d_{p}>0 \\ 0, \quad d_{r}^{B}-d_{p} \leq 0\end{array}\right.$

$d(p, B)$ represents the contrast between the depth value $d_{p}$ of pixel $p$ and the mean value $d_{r}^{B}$ (also known as the mean depth value of the sampling pixels in line $r$ ).

$d_{r}^{B}=\frac{\sum_{q \in r_{\alpha}} d_{q}}{\operatorname{col} * \alpha}$

cols is the column of the image, also known as the number of pixels of each row. $\alpha$ is the factor of the sampling point for each row. $r_{\alpha}$ is the sampling pixels in line $r$.

As shown in Fig. 3(d), only the upper half of the object is detected as salient for the first example, in which $d_{r}^{B}$ is set to the mean depth of all the pixels in line $r$; in Fig. 3(f), $d_{r}^{B}$ is set to the mean depth of $1 \%$ pixels with lower depth in line $r$, as we can see, almost the whole object is detected as salient for the first example, meanwhile, the salient regions mistakenly detected in the second example can be removed by thresholding segmentation.

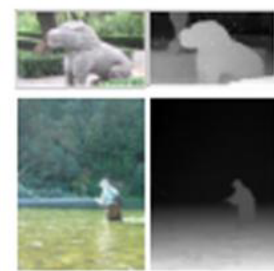

(a)

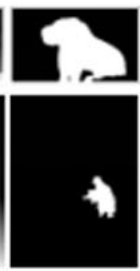

(c)

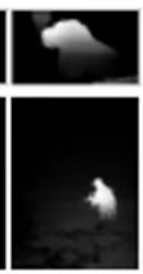

(d)

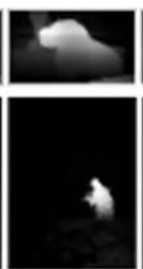

(e)

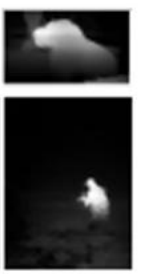

(f)
Fig. 3 Saliency detection in horizontal direction. (a) Color images, (b) Depth images, (c) Ground Truth, (d) $\alpha=1$, (e) $\alpha=0.1$, (f) $\alpha=0.01$.

\subsection{Final saliency map}

The saliency value of region $R_{i}$ is the product of global and local selective difference, as shown in equation (7), which is then normalized to $[0,255]$ and assigned to each pixel in region $R_{i}$. We can obtain the initial saliency map after completing all the sub-regions in a depth image.

$S_{s d}\left(R_{i}\right)=S_{G}\left(R_{i}\right) S_{L}\left(R_{i}\right)$

Finally, center prior is used to refine the initial saliency map, which refers to the characteristic that salient objects tend to live in the center of the image. A two-dimensional Gaussian weighted filter is presented here to obtain the final saliency map.

\section{Experiments}

In this paper, we have evaluated the results of our approach on the publicly available data set NJUDS2000 provided by Ran et al.[17], as well as we known, the data set is the largest of this kind, and has ground truth in the form of accurate human-marked labels for salient regions.

We employ the precision-recall curve to evaluate the performance of our method, which is widely used in saliency map comparison. Specifically, we obtain a binary image from each saliency map using a gradually increasing threshold from 0 to 255, and then compare with the ground truth salient object mask to get the precision and recall. We compare the proposed saliency method with 2 advanced 2D saliency detection methods: RC[3], PCA[21]; And 2 authoritative detection methods based on depth information, including ACSD[17,18] and SS[16]. The algorithm we proposed is implemented by $\mathrm{C}++$ programming, the results of other methods are compiled by the author's source code, except SS. Besides, Cheng's evaluation code [3] is used here to get the precision-recall curves of different methods.

\subsection{Experimental Setup}

The number of super-pixels in image segmentation is taken as the length of the image diagonal. The radius $\sigma$ of Gaussian template is set to 7. In saliency detection based on regional contrast, the neighborhood radius $L$ is set to onethird of the length of image diagonal, and the weight of spatial distance $\sigma^{2}$ is set to 0.4 . In saliency detection in horizontal direction, the factor of the sampling point $\alpha$ is set to 0.01 . To optimize the initial saliency map, the twodimensional Gaussian weighted filter $G\left(x, y, \sigma_{x}, \sigma_{y}\right)$ is used to optimize the initial saliency map, in which $\sigma_{x}, \sigma_{y}$ are respectively corresponding to the half-height and halfwidth of images.

\subsection{Results and Discussion}

We show the saliency comparison in Fig. 5-7(at the end of this paper), and the precision-recall curves are given in Fig. 4. As shown in Fig. 5, almost all the depth-aware saliency methods perform better than the 2D saliency methods. An exception is SS in Fig. 6, which is based on depth 
information. The background regions mistakenly detected as salient due to it's absolute value in the local contrast. Our method succeeded in surpressing the saliency of the ground area in Fig. 5 and Fig. 6. As shown in Fig. 5 and Fig. 6, ACSD performs the most close to our method as it takes center and depth priors into consideration, but it fails to distinguish the salient objects and the background when there are background regions at the bottom of the image. The precision of SS not exceed 0.6, while ACSD and our method both with priors reaches a precision far more than 0.8 . An explanation is that the data set for comparison containing too much images like this in Fig. 6. As we can see, the precision of ACSD without priors almost drop to 0.7 , in contrast, our method without center prior remains high in precision value. This suggests that priors do make a difference. In 2D saliency methods, RC nearly reaches 0.8 . The limitation of our method is that the lower half of salient objects might be lost, which can be seen in Fig. 6.

We implement our method in $C++$ and MATLAB $\mathrm{R} 2010 \mathrm{~b}$, and test a machine with a $3.30 \mathrm{GHz}$ Intel Core i5$4590 \mathrm{CPU}$ and 8GB memory. The running time is $0.431 \mathrm{~s}$ for a $499 * 372$ image.

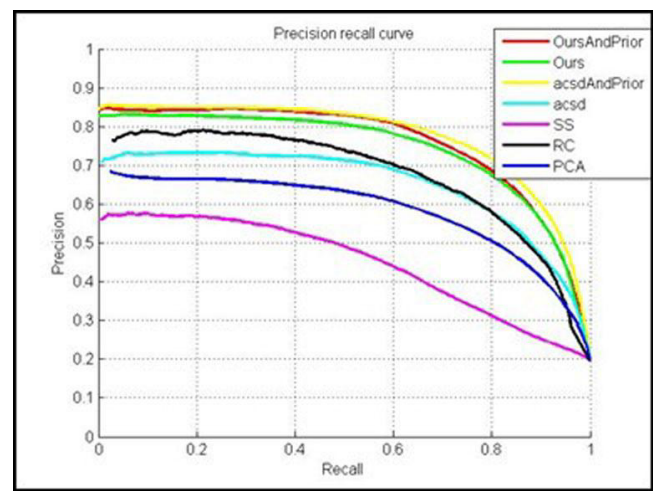

Fig. 4 Precision-recall curves of different methods.

\section{Conclusion}

In this paper, we present a new depth-aware saliency method based on selective difference. We measure the saliency of a region based on regional contrast and consider the depth difference in horizontal direction, and the validity of depth information is proved in saliency detection. However, as shown in Fig. 7, it's difficult to detect salient objects for all the 3D methods, due to the poor quality of depth images. Interestingly, the 2D saliency method get better results in Fig. 7. The problem is hoped to be solved with the improvement of depth acquisition technology, or we can try to solve the problem by combining the existing 2D saliency methods. We believe the proposed saliency methods can supply a powerful prediction in efficient object detection, reliable image recognition and classification, leading to improved image retrieval.

\section{References}

[1] L. Itti. Visual salience. Scholarpedi., 2007, 2(9):3327.

[2] Y. Fang, Z. Wang, W. Lin, and Z. Fang. Video saliency incorporating spatiotemporal cues and uncertainly weighting. IEEE Trans. Image Process., 2014, 23(9): 3910-3921.

[3] M. M. Cheng, G.-X. Zhang, N. J. Mitra, X. Huang, and S.-M.Hu. Global contrast based salient region detection. in Prol. IEEE Conf. Comput. Vis. Pattern Recog (CVPR)., 2011, pp. 409-416.

[4] L. Itti, C. Koch, and E. Niebur. A model for saliencybased visual attention for rapid scene analysis. IEEE Trans. Pattern Anal. Mach. Tntell., 1998, 20(11): 12541259.

[5] L. Zhou, Z. Yang, Q. Yuan, Z. Zhou, and D. Hu. Salient region detection via integrating diffusion-based compactness and local contrast. IEEE Trans. Image Process., 2015, 24(11): 3308-3320.

[6] M. M. Cheng, N. J. Mitra, X. Huang, P. H. S. Torr, and S. M. Hu. Global contrast based salient region detection. IEEE Trans. Pattern Anal. Mach. Intell., 2015, 37(3): 569-582.

[7] J. Sun, H. Lu, and X. Liu. Saliency region detection based on Markov absorption probabilities. IEEE Trans. Image Process. Lett., 2015, 24(5): 1639-1649.

[8] X. Wei, Z. Tao, C. Zhang, and X. Cao. Structured saliency fusion based on Dempster-Shafer theory. IEEE Signal Process. Lett., 2015,22(9): 1345-1349.

[9] C. Yang, L. Zhang, H. Lu, X. Ruan, and M. H.Yang. Saliency detection via graph-based manifold ranking. in Proc. IEEE Conf. Comput. Vis. Pattern Recog . (CVPR), 2013, pp. 3166-3173.

[10] Y. Fang, J. Wang, M. Narwaria, P. Le Callet, and W. Lin. Saliency detection for stereoscopic images. IEEE Trans. Image Process., 2014, 23(6): 2625-2636.

[11] C. Lang, T. V. Nguyen, H. Katti, K. Yadati, M. Kankanhalli, and S. Yan. Depth Matters: Influence of Depth Cues on Visual Saliency. In ECCV, 2012.

[12] J. Ren, X. Gong, L. Yu, W. Zhou, and M. Yang. Exploiting global priors for RGB-D Saliency detection. In CVPRW, 2015, pp. 25-32.

[13] H. Peng, B .Li, W. Xiong, W. Hu, and R. Ji. RGBD Salient Object Detection: A Benchmark and Algorithms. In ECCV, 2014.

[14] A. Ciptadi, T. Hermans, and J. M. Rehg. An In Depth View of Saliency. In BMVC, 2013.

[15] K. Desingh, K. M. Krishna, D. Rajan, and C. V. Jawahar. Depth Really Matters: Improving Visual Salient Region Detection with Depth. In BMVC, 2013.

[16] Yuzhen Niu, Yujie Geng, Xueqing Li, and Feng Liu. Leveraging stereopsis for saliency analysis. in IEEE Conference on Computer Vision and Pattern Recognition. IEEE, 2012, pp. 454-461.

[17] R. Ju, L. Ge, W. Geng, T. Ren, and G. Wu. Depth saliency based on anisotropic center-surround difference. ICIP, 2014. 
[18] R. Ju, Y. Liu, T. Ren, L. Ge, and G. Wu. Depth-aware salient object detection using anisotropic centersurround difference. Signal Process. Image Commun., 2015, 38(10): 115-126.

[19] Deqing Sun, Stefan Roth, and Michael J Black. Secrects of optical flow estimation and their principles. in IEEE Conference on Computer Vision and Pattern Recognition. IEEE, 2010, pp. 2432-2439.
[20] Radhakrishna Achanta, Appu Shaji, Kevin Smith, Aurelien Lucchi, Pascal Fua, and Sabine Susstrunk. Slic superpixels compared to state-of-the-art superpixel methods. IEEE Transactions on Pattern Analysis and Machine Intelligence., 2012, 34(10):2274-2282.

[21] R. Margolin, A. Tal, L. Zelnik-Manor, What makes a patch distinct? IEEE Conference on Computer Vision and Pattern Recognition, 2013, pp. 1139-1146. 


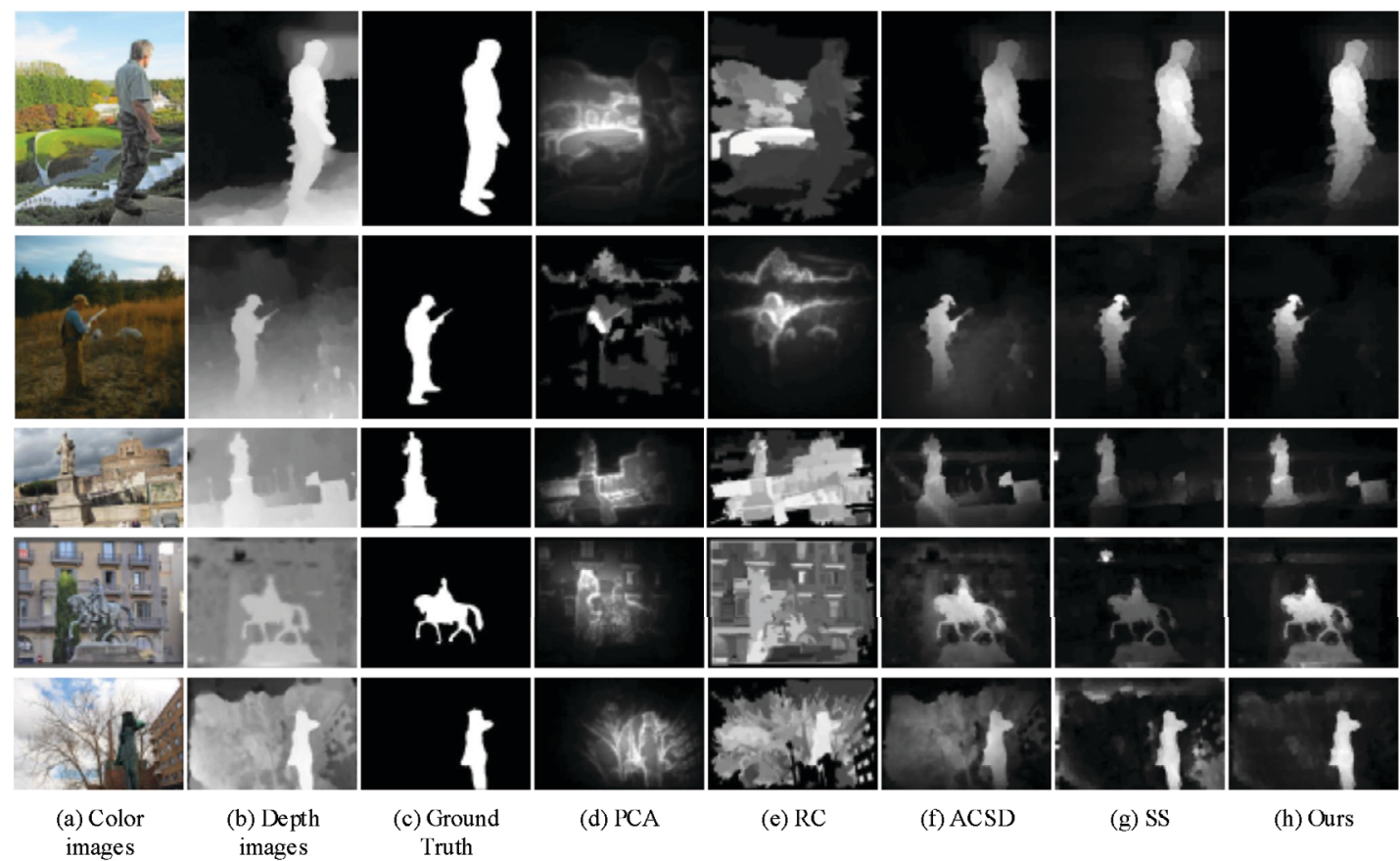

Fig. 5 Saliency comparison of different methods

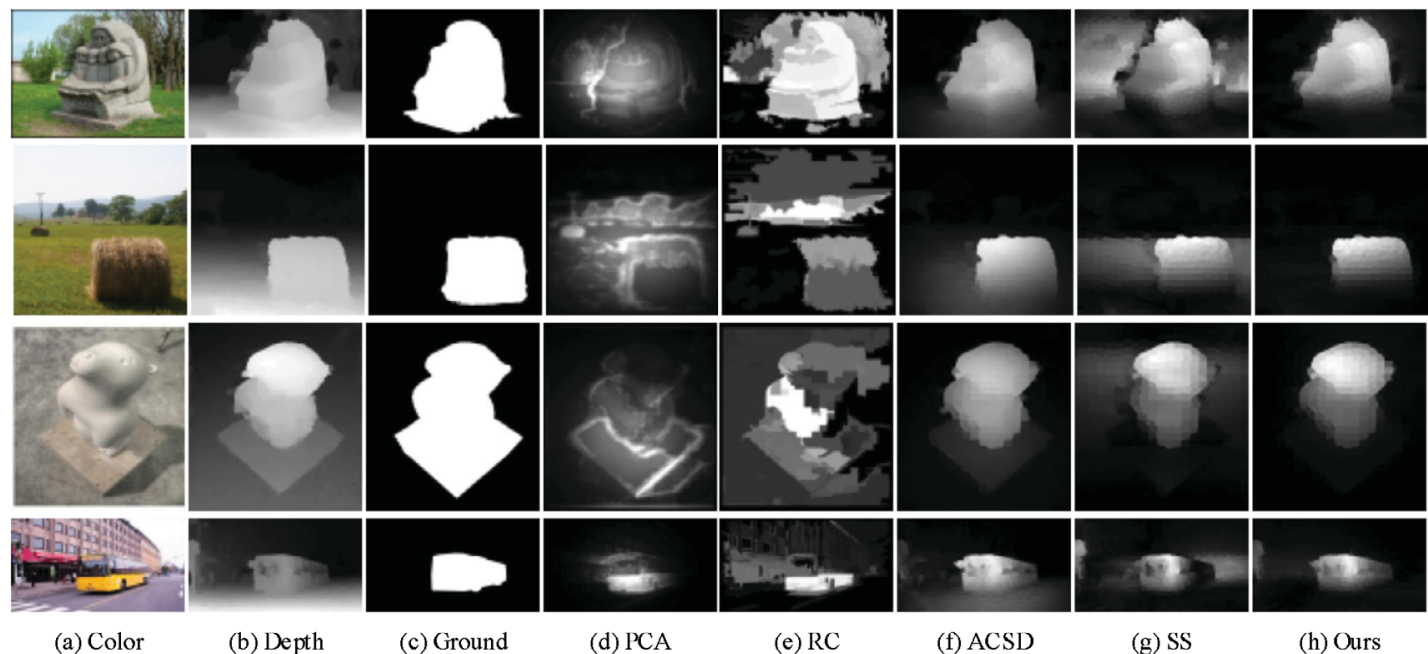

Fig. 6 Saliency comparison of different methods

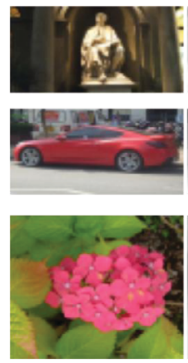

(a) Color images

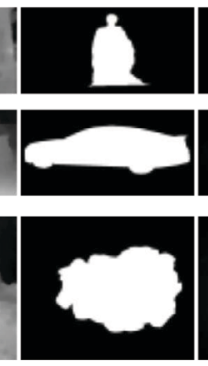

(c) Ground Truth

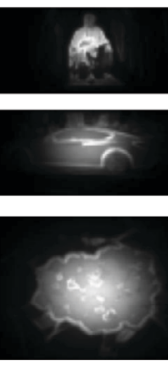

(d) PCA

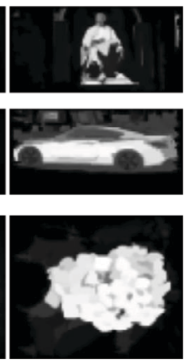

(e) $\mathrm{RC}$
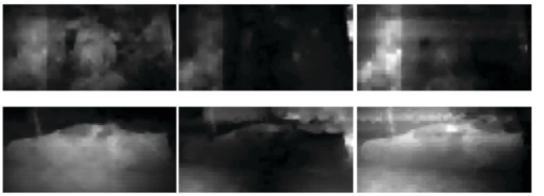

Fig. 7 Failure cases of depth based saliency detection. 